International Mathematical Forum, 1, 2006, no. 26, 1273 - 1284

\title{
Plemwanal:
}

\section{A Communicating Computing Mathematics Generator Type}

\author{
Fevzi Ünlü \\ Department of Mathematics, Yasar University, \\ Bornova, Izmir, Turkey \\ e-mail: fevzi.unlu@yasar.edu.tr and fevziunlu@ttnet.net.tr
}

\begin{abstract}
[1] Has introduced the notion of $I @ I$. [2-10] have developed, studied and introduced the fundamentals of computing mathematics on 1) Prüfer's type I@I clusters; 2) the notion of knowledge based object $K B O ; 3)$ integration and derivation of $K B O ; 4)$ us-crop; 5) us-culture; 6) tuze-channeled KBO for DCP; 7) linear-circular convoluted us-crop I@I KBO cluster generating us-crop; 8) us-crop based compact plasma memory; and 9) us-crop based plemal. Discrete mathematical backgrounds and point set topology backgrounds can be found in [11, 12]. Combinatorial mathematical backgrounds can be found in [13]. This paper develops, studies and introduces some fundamentals of a formal language type that generates a communicating computer mathematics type virtual machine KBO and its remote programming style on us-crop based compact plasma memory by ten definitions, two corollary and one theorem. It is called as plemwanal in this paper. Author claims that plemwanal is a very special communicating computur mathematics type virtual machine $\mathrm{KBO}$ and its remote programming style generating language type. It is produced as an extension of plemal for obtaining an artificial intelligently communicating numerical words with antennae. These numerical words with antennae are communicating themselves by broadcasting and intercepting information in the execution time of its substructures. Eeach token numerical word with antennae in each substructure has wireless communication property with each other and with its environment. It is designed for generating remote, parallel, sequential, mobile, distributed, etc. type us-crop based compact I@I KBO cluster plasma memory programming in classical or quantum information processing environment. After plemwanal there is going to be a new
\end{abstract}


computing mathematics type. The author calls it as "Communicating Computer Mathematics."

Mathematics Subject Classification: 00A05, 00A08, 00A35, 00A71, 03F50, 44A55, 47A65, 68P20, 68Q10, 68Q65, 68Q85, 94A05, 94A16.

Keywords: KBO, us-crop I@I KBO cluster, us-crop I@I cluster memory type, formal grammar, formal language, remote programming style, virtual machine $\mathrm{KBO}$, communicating computer mathematics.

\section{Introduction}

[1] has introduced the notion of $I @ I$. [2-10] have developed, studied and introduced the fundamentals of computer mathematics on 1) Prüfer's type $I @ I$ clusters; 2) the notion of knowledge based object $-K B O ; 3)$ integration and derivation of $K B O$; 4) us-crop; 5) us-culture; 6) tuze-channeled KBO for DCP; 7) linear-circular convoluted us-crop I@I KBO cluster generating us-crop; 8) us-crop based compact plem memory; 9) us-crop based plemal. Discrete mathematical backgrounds and point set topology backgrounds can be found in $[11,12]$. Combinatorial mathematical backgrounds can be found in [13]. This paper develops, studies and introduces some fundamentals of a formal language type that generates a communicating computer mathematics type virtual machine KBO and its remote programming style on us-crop based compact plem memory by ten definitions, two corollary and one theorem. It is called as plemwanal in this paper. Author claims that plemwanal is a very special communicating computer mathematics type virtual machine $\mathrm{KBO}$ and its remote programming style generating language type. It is produced as an extension of plemal for obtaining an artificial intelligently communicating numerical words with antennae. These numerical words with antennae are communicating themselves by broadcasting and intercepting information in the execution time of its substructures. Each token numerical word with antennae in each substructure has wireless communication property with each other and with its environment. It is designed for generating remote, parallel, sequential, mobile, distributed, etc. type us-crop based compact I@I KBO cluster plem memory programming in classical or quantum information processing environment. After plemwanal there is going to be a new computing mathematics type. The author calls it as "Communicating Computer Mathematics." 


\section{$2 \quad$ Preliminary Notes}

[1-10] introduce the fundamentals of computing mathematics of data structures in/on us-crop and syntax, semantics and pragmatics of plemal. Those structures are currently adapted into plemwanal for current use and making it isomorph to plemal. In this section, therefore, the preliminary notions and tools which are necessary for generating plemwanal will be introduced. The needed discrete mathematical backgrounds and point set topology backgrounds can be found in $[11,12]$. Combinatorial mathematical backgrounds can be found in $[13]$.

\subsection{Plem and Data Structures on Plem}

Definition 1 Let 1) "plem" be a brief vocabulary for programmable linear

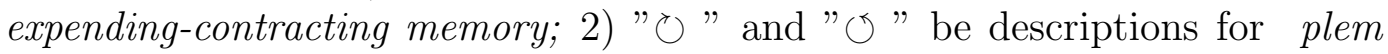
icons for representing u-crop and s-crop; 3) "KBO" be a brief vocabulary for Knowledge Based Object. 4) "(" be a broadcasting antenna icon; 5) "〉" be an intercepting antenna icon; and 6) " $"$ " be a formal KBO type; 7) " $\mathrm{Z}^{+}$" be the set of positive integers; 8) "«" be a generalized assignment operator; 9) "|" be a logical $O R$ operator in the descriptive language (in an $\alpha$-language type); 10) $\mathrm{r}_{i}$ be a proper KBO register type that it is indexed by $i \in \mathrm{Z}^{+}$;11) plemwan be a brief vocabulary for "programmable linear expanding-contracting memory with antennae network." Then 1) $\langle\zeta\rangle$ is called a $K B O \zeta$ with a pair of broadcasting and intercepting antennae; 2) $\mathrm{r}_{u}$ is called an u-plem register, if $\left.\mathrm{r}_{u}=\circlearrowright ; 3\right) \mathrm{r}_{s}$ is called an s-plem register, if $\mathrm{r}_{s}=\circlearrowleft$; 4) $\mathrm{r}$ is called a plem register; if $\mathrm{r}$ takes the value of $\left.\mathrm{r}_{u} \mid \mathrm{r}_{s} .5\right)\left\langle\mathrm{r}_{1}^{\diamond}, \mathrm{r}_{2}^{\diamond}, \mathrm{r}_{3}^{\diamond}: \mathrm{r}_{u}\right\rangle=\left\langle\mathrm{r}_{3}, \mathrm{r}_{2}, \mathrm{r}_{1}: \mathrm{r}_{u}\right\rangle$ is called an u-plemwan token, if $\left\langle\mathrm{r}_{3}, \mathrm{r}_{2}, \mathrm{r}_{1}: \mathrm{r}_{u}\right\rangle=\left\langle\begin{array}{lll}r_{3} & r_{u} & r_{1} \\ & r_{2}\end{array}\right\rangle=\left\langle\begin{array}{lll}r_{3} & \circlearrowright & r_{1} \\ & r_{2}\end{array}\right\rangle$. Where (i) $\mathrm{r}_{1} \longleftarrow v_{1}$, $v_{1} \in[\mathrm{p}+1]=\{0,1, \ldots, \mathrm{p}\}, \mathrm{p} \in \mathrm{Z}^{+} ;$(ii) $\mathrm{r}_{2} \longleftarrow v_{2}, v_{2} \in[\mathrm{x}+1]=\{0,1, \ldots$, $\mathrm{x}\}, \mathrm{x} \in \AA$, where $\AA$ is a letteral alphabet; (iii) $\mathrm{r}_{3} \longleftarrow v_{3}, v_{3} \in[\mathrm{m}+1]=\{0,1$, $\left.\ldots, \mathrm{m}\}, \mathrm{m} \in \mathrm{Z}^{+} .6\right)\left\langle\mathrm{r}_{1}, \mathrm{r}_{2}, \mathrm{r}_{3}: \mathrm{r}_{s}\right\rangle$ is called an s-plemwan token, if $\left\langle\mathrm{r}_{1}, \mathrm{r}_{2}, \mathrm{r}_{3}\right.$ $\left.: \mathrm{r}_{s}\right\rangle=\left\langle\begin{array}{lll}r_{1} & r_{s} & r_{3} \\ & r_{2} & \end{array}\right\rangle=\left\langle\begin{array}{lll}r_{1} & \circlearrowleft & r_{3} \\ & r_{2} & \end{array}\right\rangle$. Where (i) $\mathrm{r}_{1} \longleftarrow v_{1}, v_{1} \in[\mathrm{q}+1]=\{0$, $1, \ldots, \mathrm{q}\}, \mathrm{q} \in \mathrm{Z}^{+}$; (ii) $\mathrm{r}_{2} \longleftarrow v_{2}, v_{2} \in[\mathrm{y}+1]=\{0,1, \ldots, \mathrm{y}\}, \mathrm{y} \in \ddot{A}$, where $\ddot{\mathrm{A}}$ is a letteral alphabet; (iii) $\mathrm{r}_{3} \longleftrightarrow v_{3}, v_{3} \in[\mathrm{n}+1]=\{0,1, \ldots, \mathrm{n}\}, \mathrm{n} \in \mathrm{Z}^{+}$;

Corollary $1 \mathrm{r} \longleftarrow \mathrm{r}_{u} \mid \mathrm{r}_{s}$ implies $\left\langle\mathrm{r}_{1}, \mathrm{r}_{2}, \mathrm{r}_{3}: \mathrm{r}\right\rangle \longleftarrow\left\langle\mathrm{r}_{3}, \mathrm{r}_{2}, \mathrm{r}_{1}: \mathrm{r}_{u}\right\rangle \mid\left\langle\mathrm{r}_{1}, \mathrm{r}_{2}\right.$, $\left.\mathrm{r}_{3}: \mathrm{r}_{s}\right\rangle$.

Proof Let 1$) \mathrm{r}_{u}$ be an u-plem register; 2) $\mathrm{r}_{s}$ be an s-plem register. Then $\mathrm{r} \longleftarrow \mathrm{r}_{u} \mid \mathrm{r}_{s}$ implies that $\left\langle\mathrm{r}_{1}, \mathrm{r}_{2}, \mathrm{r}_{3}: \mathrm{r}\right\rangle \longleftarrow\left\langle\mathrm{r}_{3}, \mathrm{r}_{2}, \mathrm{r}_{1}: \mathrm{r}_{u}\right\rangle \mid\left\langle\mathrm{r}_{1}, \mathrm{r}_{2}, \mathrm{r}_{3}: \mathrm{r}_{s}\right\rangle$. 


\subsection{Plemwana: Alphabet of Plemwanal}

Definition 2 Let "FTD" be a brief vocabulary for "formal technology dependent". 1) A mod p enforced(registered by an available formal technology), and m-ary negative u-crop I@I KBO cluster memory with a pair of broadcasting and intercepting antennae is called as an m-ary negative FTD plem memory type with a pair of broadcasting and intercepting antennae. It is represented by $\left\langle\mathrm{X}_{-}\right\rangle=\langle p \circlearrowright m\rangle \Longleftrightarrow[-\mathrm{m} ;-0]\left\langle\mathrm{X}_{-}\right\rangle @\left\langle\mathrm{X}_{+}\right\rangle[+\phi ;+\phi] \bmod \mathrm{p}=\operatorname{array}[-\mathrm{m} \ldots$ $-0]$ of $\left(\left\langle\mathrm{X}_{-}\right\rangle \bmod \mathrm{p},\left\langle\mathrm{X}_{+}\right\rangle \bmod \phi\right)$. 2) A $\bmod \mathrm{q}$ enforced, and n-ary positive s-crop I@I KBO cluster memory is called an m-ary positive plem memory type with a pair of broadcasting and intercepting antennae. It is represented by $\mathrm{X}_{+}$ $=\langle n \circlearrowleft q\rangle \Longleftrightarrow[-\phi ;-\phi]\left\langle\mathrm{X}_{-}\right\rangle @\left\langle\mathrm{X}_{-}\right\rangle[+0 ;+n] \bmod \mathrm{q}=\operatorname{array}[+0 . .+\mathrm{n}]$ of $\left(\left\langle\mathrm{X}_{-}\right\rangle\right.$ $\left.\left.\bmod \phi,\left\langle\mathrm{X}_{+}\right\rangle \bmod q\right) .3\right) \Omega=\{\langle p \circlearrowright m\rangle,\langle n \circlearrowleft q\rangle\}$ is called a plemwana type KBO for $\mathrm{p}, \mathrm{q} \geq 2$ and $\mathrm{p}, \mathrm{m}, \mathrm{n}, \mathrm{q} \in \mathrm{N}$. Where plemwana is a brief vocabulary for "alphabet of programmable linearly-extending-contracting-plema memory with antenna networks."

\subsection{Plemwanal}

Definition 3 Let $p, q \geq 2 ; m, n, p$ and $q \in N$ and $N$ be the set of natural numbers. Let $\Omega=\{\langle m \circlearrowright p\rangle,\langle q \circlearrowleft n\rangle\}$ be a plemwana. 1) $\hat{\mathrm{G}}=\left\{\mathrm{R}_{0}=\Omega^{0}=\{\right.$ $\left.\varepsilon\}, \mathrm{R}_{1}: \Omega^{1}=\Omega, \mathrm{R}_{2}=\Omega^{t}=\left\{\mathrm{x} \circ \mathrm{y}=\mathrm{xy}: \mathrm{x} \in \Omega, \mathrm{y} \in \Omega^{t-1}, t \geq 2, \mathrm{t} \in N\right\}\right\}$ is called a recursive formal grammar of plemwanal on the plemwana $\Omega$ under concatenation operator o. 2) $\Omega^{+}=\cup_{t=1}^{\infty} \Omega^{t}=\cup_{t \in Z^{+}}^{\infty} \Omega^{t}$ is called plemwana ${ }^{+}$. 3) $\Omega^{*}=\cup_{t=0}^{\infty} \Omega^{t}=\cup_{t \in N}^{\infty} \Omega^{t}$ is called plemwana* ${ }^{*}$ 4) Each string element in plemwana* $^{*}$ is called a plemwanal word. 5) Any subset of a plemwana* is called a plemwanal. Plemwanal is a new constructed vocabulary. It stands for the family of languages generated on a plemwana $\Omega$ by the recursive plemwana grammar $\hat{\mathrm{G}}$. It includes empty subset $\phi$, which is empty plemwanal.

Corollary 2 (a) Plemwanal is a family of formal language on plemwana $\Omega=\{\langle m \circlearrowright p\rangle,\langle q \circlearrowleft n\rangle\}$. (b) Each plemwanal is isomorph to plemal on plema $\Sigma=\{m \circlearrowright p, q \circlearrowleft n\}$.

Proof (a) Plemwanal is a family of formal language due to its definition. (b) Each plemwanal is isomorph to a plemal because only the terminal symbols in their alphabet plemwana and plema are different from each other.

\section{Plemwanal KBO Configurations}

Definition 4 (a) Let $\mathrm{x}$ be a plemwan. $\mathrm{x} \longleftarrow\langle m \circlearrowright p\rangle \mid\langle n \circlearrowleft q\rangle$; $\mathrm{p}, p \geq 2, \mathrm{p}, \mathrm{q}$, $\mathrm{n}, \mathrm{m} \in \mathrm{N}$; Each element of $\mathrm{x}$ with different combinatorial values assigned for $\mathrm{p}, \mathrm{q}, \mathrm{n}$, and $\mathrm{m}$ is called a plemwan configuration. (b) Let y be a plemwanal word. Each element of $y \in \Omega^{*}$ with different combinatorial values assigned 
for $\mathrm{p}, \mathrm{q}, \mathrm{n}$, and $\mathrm{m}$ is called a plemwanal word configuration. (c) Let $\mathrm{z}$ be a plemwanal L. Each element of $z \subseteq \Omega^{*}$ with different values assigned for $\mathrm{p}, \mathrm{q}$, $\mathrm{n}$, and $\mathrm{m}$ is called a plemwanal configuration.

\subsection{General Plemwanal KBO Structure Types}

Definition 5 The followings are called general configuration KBO types:

01) $[-u ;-0]=[-u, \ldots,-0], \mathrm{u} \in \mathrm{N}$.

02) $[+0 ;+s]=[+0, \ldots,+s], \mathrm{s} \in \mathrm{N}$.

03) $\left[\phi_{-u} ; \phi_{-0}\right]=\left[\phi_{-u}, \ldots, \phi_{-0}\right]=-\Phi$, where $-\Phi$ is called KBO of negative emptiness, if $\Phi_{-i} \longleftarrow \Phi$ for $\mathrm{i} \in[-\mathrm{u},-0]$.

04) $\left[\phi_{+0} ; \phi_{+s}\right]=\left[\phi_{+0}, \ldots, \phi_{+s}\right]=+\Phi$, where $+\Phi$ is called $\mathrm{KBO}$ of positive emptiness, if $\Phi_{+i} \longleftarrow \Phi$ for $\mathrm{i} \in[+0,+\mathrm{s}]$.

05) $\mathrm{u}$-crop $=$ a set of spikes indexed by $[-u ;-0]=[-u, \ldots,-0], \mathrm{u} \in \mathrm{N}$.

06) $\mathrm{s}$-crop $=$ a set of spikes indexed by $[+0 ;+s]=[+0, \ldots,+s], \mathrm{s} \in \mathrm{N}$.

07) $m \circlearrowright p=\mathrm{u}$-crop token of the spike-values that they take their values in $\bmod p$, where $p \geq 2, \mathrm{p}, \mathrm{u} \in \mathrm{N}$.

08) $q \circlearrowleft n=$ s-crop token of the spike-values that they take their values in $\bmod q$, where $q \geq 2, \mathrm{q}, \mathrm{u} \in \mathrm{N}$.

09) us-crop $=$ a set of spikes that they are indexed by $[-u ;-0] \cup[+0$; $+s]$, where $\mathrm{u}, \mathrm{s} \in \mathrm{N}$.

10) $\mathrm{u}$-culture $=\mathrm{a}$ set of $\mathrm{u}$-crops that they are indexed by $\left[-\mathrm{u}_{i} ;-0\right]$, where $\mathrm{u}_{i},{ }_{i} \in \mathrm{N}$.

11) s-culture $=$ a set of s-crop that they are indexed by $\left[+0 ;+s_{j}\right]$, where $\mathbf{s}_{j}, j \in \mathrm{N}$.

12) $\mathrm{us}_{\text {-culture }}=\mathrm{a}$ set of us-crops that they are indexed by $\left[-\mathrm{u}_{i} ;-0\right] \cup$ $\left[+0 ;+s_{j}\right]$, where $\mathrm{u}_{i}, \mathrm{~s}_{j},{ }_{i},{ }_{j} \in \mathrm{N}$.

13) plem $\longleftrightarrow m \circlearrowright p \mid q \circlearrowleft n$; where $p, q \geq 2, p, q, n, m \in \mathrm{N}$.

14) plema $=\{m \circlearrowright p, q \circlearrowleft n\}$; where $p, q \geq 2, p, q, n, m \in \mathrm{N}$.

15) plemal $=$ a subset of plema*.

16) plemwan $\longleftarrow\langle m \circlearrowright p\rangle \mid\langle n \circlearrowleft q\rangle$; where $p, p \geq 2, p, q, n, m \in \mathrm{N}$. N.

17) plemwana $\longleftarrow\{\langle m \circlearrowright p\rangle,\langle n \circlearrowleft q\rangle\}$; where $p, p \geq 2, p, q, n, m \in$

18) plemwanal $=$ a subset of plemwana*.

Where ${ }^{*}$ is Kleene's Closure operator. It produces all words(or strings) from a given alphabet.

\subsection{Variable KBO Structure types}

Definition 6 The followings are called variable-configuration KBO structure types.

1) $\delta=$ a character type of $\mathrm{a}|\mathrm{b}| \ldots|\mathrm{x}| \mathrm{y} \mid \mathrm{z}$. 
2) $\Delta=$ a character type of A $|\mathrm{B}| \ldots|\mathrm{X}| \mathrm{Y} \mid \mathrm{Z}$.

3) $\Sigma$ : Plema.

4) $\Omega$ : Plemwana.

5) $\delta$ : the set of plemwanal-word variable.

6) $\Delta$ : the set of plemwanal variable.

7) L : formal language.

\subsection{Constant KBO Structure Types}

Definition 7 The followings are called constant-configuration KBO types.

1) $\mathrm{Z}^{+}$: The set of positive numbers.

2) $\mathrm{N}$ : The set of natural numbers.

3) $[\mathrm{m}]$ : The set of mod $\mathrm{m}$ numerals. That is $[\mathrm{m}]=\{0,1,2, \ldots, \mathrm{m}-1\}$.

\subsection{Structured Construction KBO Types}

Definition 8 The followings are called construction-configuration KBO structure types:

01) $\Omega^{1}=\Omega$.

02) $\Omega^{n+1}=\left\{\right.$ xoy $\left.=\mathrm{xy}: \mathrm{x} \in \Omega, \mathrm{y} \in \Omega^{n}\right\}$, where $\mathrm{xy}=\mathrm{x} \circ \mathrm{y}$ denotes the concatenation of $\mathrm{x}$ and $\mathrm{y}$.

03) $\Omega^{+}=$Plus closure of $\Omega$

04) $\Omega^{*}=$ Kleen's closure of $\Omega$.

05) $\Omega^{*}=\{\varepsilon\} \cup \Omega^{+}$.

06) $\mathrm{L} \subseteq \Omega^{*}$.

07) $\mathrm{L}^{0}=\{\varepsilon\}$.

08) $\mathrm{L}^{1}=\mathrm{L}$.

09) $L^{n+1}=\left\{\right.$ xoy $\left.=\mathrm{xy}: \mathrm{x} \in L, \mathrm{y} \in L^{n}\right\}$.

10) $L^{+}=$Plus closure of $L$.

11) $L^{*}=$ Kleen's closure of $\mathrm{L}$.

12) $L^{*}=\{\varepsilon\} \cup L^{+}$.

\subsubsection{Function KBO Types}

Definition 9 Let K be a parameteric list of KBO's or KBO clusters, and M be a KBO-plem cluster memory in a given universal KBO cluster memory Ü. Let viros be a brief vocabulary for "virtual robotic operating system." The followings are called viros-function configurational KBO types:

01 Begin is a delimeter for the begining of a new action in the current agorithm.

02 Reserve $(\mathrm{K}: \mathbf{M})$ is a viros-function type that it reserves a KBO K in the $\mathrm{M}^{\text {th }}$ partition of $\ddot{\mathrm{U}}$. 
$03 \mathbf{I n j e c t}(\mathbf{K}: \mathbf{M})$ is a viros-function type that it injects a KBO K into $\mathrm{M}^{\text {th }}$ partition of $\ddot{\mathrm{U}}$.

04 Point $(\mathbf{K}: \mathbf{M} ; \mathbf{x})$ is a viros-function type that it points a $\mathrm{KBO} \mathrm{K}$ in the $\mathrm{M}^{\text {th }}$ partition of $\ddot{\mathrm{U}}$ by a beam type $\mathrm{x}$.

05 ShowKonfigurationof(K: $\mathbf{M})$ is a viros-function type that it shows the configuration of $\mathrm{KBO} \mathbf{K}$ in the $\mathrm{M}^{\text {th }}$ partition of $\ddot{\mathrm{U}}$.

06 ShowDesignof $(\mathbf{K}: \mathbf{M})$ is a viros-function type that it shows design of $\mathrm{KBO} \mathrm{K}$ in the $\mathrm{M}^{\text {th }}$ partition of $\ddot{\mathrm{U}}$.

07 ShowBeamof $(\mathbf{K}: \mathbf{M})$ is a viros-function type that it shows beam of $\mathrm{KBO} \mathrm{K}$ in the $\mathrm{M}^{\text {th }}$ partition of $\ddot{\mathrm{U}}$.

08 ShowusCultureof $(\mathbf{K}: \mathbf{M})$ is a viros-function type that it shows the us-culture of KBO K in the $\mathrm{M}^{\text {th }}$ partition of $\ddot{\mathrm{U}}$.

09 ShowLoadof $(\mathbf{K}: \mathbf{M})$ is a viros-function type that it shows the loads of $\mathrm{KBO} \mathrm{K}$ in the $\mathrm{M}^{\text {th }}$ partition of $\ddot{\mathrm{U}}$.

10 FixLoad(K: $\mathbf{M})$ is a viros-function type that it fixes the load of KBO $\mathrm{K}$ in $\mathrm{M}$.

11 RealizeLoad(K: $\mathbf{M})$ is a viros-function type that it realizes the fixed load of $\mathrm{KBO} \mathrm{K}$ in $\mathrm{M}$.

12 ShowFixedLoad(K: $\mathbf{M})$ is a viros-function type that it showes the fixed loads of $\mathrm{KBO} \mathrm{K}$ in the $\mathrm{M}^{\text {th }}$ partition of $\ddot{\mathrm{U}}$.

13 BeamFor Load(A, B:M; x, y) is a viros-function type that it loads KBO $\mathrm{A}$ and $\mathrm{KBO} \mathrm{B}$ in $\mathrm{M}$ by the beams $\mathrm{x}$ and $\mathrm{y}$.

14 Stop stops the action of a current algorithm for a while.

15 End is delimeter for ending the action of the current algorithm.

\section{Remote Programming Style}

Definition 10 Let 1) emw be a brief vocabulary for "electoral magnetic wave." 2) $\langle\mathrm{x}\rangle$ be a remote KBO $\mathrm{x}$ with a pair of broadcasting and intercepting antennae network that they are sensitive to emw. Any programing style that programs $\mathrm{x}$ from a remote distance by using some coded(or codable) information into beam of emw(or light) by a FTD technology is called a remote programming style.

Theorem Let 1) $\mathrm{M}$ be a reserved KBO plem memory cluster in KBO $\mathrm{Q}^{\text {th }}$ partition of KBO $\ddot{\mathrm{U}}$; 2) "viros" be a brief vocabulary for describing the notion of "virtual robotic operating system;" 3) A, B, C and U be KBOclusters can be generated in $\mathrm{M}$ by injection such a way that $\mathrm{A}$ and $\mathrm{B}$ becomes to be generated in $\mathrm{C}$ and $\mathrm{C}$ becomes to be generated in $\hat{\mathrm{U}}$; 4 ) F be a virosfunction type (or class) KBO-cluster that it contains the viros-functions given in Definition 9. There is at least one remote programming style to program: (a) $\mathrm{A}$ and $\mathrm{B}$ in $\mathrm{C}$ in $\hat{\mathrm{U}}$ by a plemwan $x$; (b) $\mathrm{A}$ and $\mathrm{B}$ in $\mathrm{C}$ in $\hat{\mathrm{U}}$ by a plemwanal word $y$; (c) $\mathrm{A}$ and $\mathrm{B}$ in $\mathrm{C}$ in $\hat{\mathrm{U}}$ by a plemwanal $z$. 
Proof (a) The following Algorithm R1 is a remote programming style to program $\mathrm{A}$ and $\mathrm{B}$ in $\mathrm{C}$ in $\hat{\mathrm{U}}$ by a plemwan. (b) Algorithm R2 is a remote programming style to program $\mathrm{A}$ and $\mathrm{B}$ in $\mathrm{C}$ in $\mathrm{U}$ by a plemwanal word. (c) Algorithm R3 is a remote programming style to program $\mathrm{A}$ and $\mathrm{B}$ in $\mathrm{C}$ in $\hat{\mathrm{U}}$ by a plemwanal.

(a) Algorithm R1

Let $\mathrm{x}$ and $\mathrm{y}$ be two variables on the plemwan KBO's. Let the conditions in the hypothesis exist for the current use.

S00 Begin; $\ddot{\mathrm{U}} \longleftrightarrow \mathrm{M} ;$

S01 Reserve(M: Ü) reserves the $\mathrm{M}^{t h}$ partion of $\ddot{\mathrm{U}}$. Hence, one has

S02 Inject( $\hat{\mathbf{U}}: \mathbf{M})$ injects $\hat{\mathrm{U}}$ into $\mathrm{M}^{\text {th }}$ partition of $\ddot{\mathrm{U}}$. Hence, one has $\ddot{\mathrm{U}} \longleftrightarrow \mathrm{M} \longleftarrow \hat{\mathrm{U}} ;$

S03 Inject(C: $\hat{U})$ injects $C$ into $C^{t h}$ partition of $M$. Hence, one has $\ddot{\mathrm{U}} \hookleftarrow \mathrm{M} \longleftarrow \hat{\mathrm{U}} \longleftarrow \mathrm{C} ;$

S04 Inject (A, B: C) injects A and B into $\mathrm{C}^{\text {th }}$ partion of $\hat{\mathrm{U}}$. Hence, one has $\ddot{\mathrm{U}} \longleftarrow \mathrm{M} \longleftarrow \hat{\mathrm{U}} \longleftarrow \mathrm{C} \longleftarrow \mathrm{A}$ and $\mid$ or $\mathrm{B}$;

S05 Point $\left(A \longleftarrow x=\left\langle\mathrm{r}_{3}, \mathrm{r}_{2}, \mathrm{r}_{1}: \mathrm{r}_{u}\right\rangle\right.$ : C) points $\mathrm{A}$ by a ray of light called $\mathrm{x}$ that does carry some loaded information $\left\langle\mathrm{r}_{3}, \mathrm{r}_{2}, \mathrm{r}_{1}: \mathrm{r}_{u}\right\rangle$. Hence, one has $\ddot{\mathrm{U}} \longleftarrow \mathrm{M} \longleftarrow \hat{\mathrm{U}} \longleftarrow \mathrm{C} \longleftarrow\left(\mathrm{A} \longleftarrow \mathrm{x}=\left\langle\mathrm{r}_{3}, \mathrm{r}_{2}, \mathrm{r}_{1}: \mathrm{r}_{u}\right\rangle, \mathrm{B}\right)$;

S06 Point $\left(B \longleftarrow y=\left\langle r_{1}, r_{2}, r_{3}: r_{s}\right\rangle: C\right)$ points $B$ by a ray of light called y that does carry some loaded information $\left\langle\mathrm{r}_{1}, \mathrm{r}_{2}, \mathrm{r}_{3}: \mathrm{r}_{s}\right\rangle$. Hence, one has $\ddot{\mathrm{U}} \longleftarrow \mathrm{M} \longleftarrow \hat{\mathrm{U}} \longleftarrow \mathrm{C} \longleftarrow\left(\mathrm{A} \longleftarrow \mathrm{x}=\left\langle\mathrm{r}_{3}, \mathrm{r}_{2}, \mathrm{r}_{1}: \mathrm{r}_{u}\right\rangle, \mathrm{B} \longleftarrow \mathrm{y}=\left\langle\mathrm{r}_{1}, \mathrm{r}_{2}, \mathrm{r}_{3}: \mathrm{r}_{s}\right.\right.$ : C);

S07 ShowConfigurationof $(A, B$ : C) shows the configuration of (A, $\mathrm{B})$ in $\mathrm{C}$. Hence, one has $\mathrm{C} \longleftarrow\left(\mathrm{A} \longleftarrow \mathrm{x}=\left\langle\mathrm{r}_{3}, \mathrm{r}_{2}, \mathrm{r}_{1}: \mathrm{r}_{u}\right\rangle, \mathrm{B} \longleftarrow \mathrm{y}=\left\langle\mathrm{r}_{1}\right.\right.$,

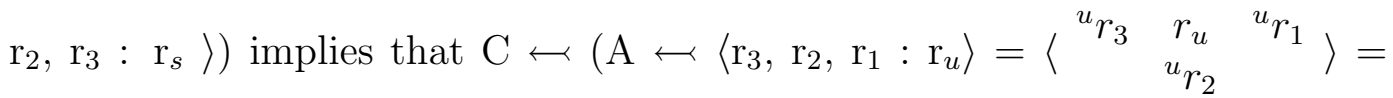

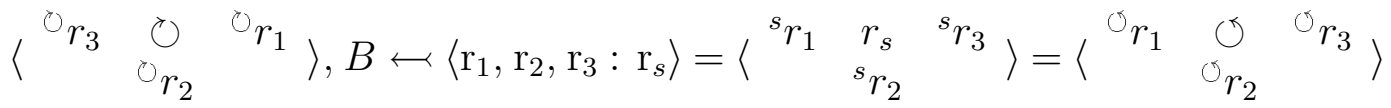
: C).

Where (i) ${ }^{u} \mathrm{r}_{1} \longleftrightarrow v_{1}, v_{1} \in[\mathrm{p}+1]=\{0,1, \ldots, \mathrm{p}\}, \mathrm{p} \in \mathrm{Z}^{+} ;$(ii) ${ }^{u} \mathrm{r}_{2} \longleftrightarrow v_{2}$, $v_{2} \in[\mathrm{x}+1]=\{0,1, \ldots, \mathrm{x}\}, \mathrm{x} \in \AA$, where $\AA$ is a letteral alphabet; (iii) ${ }^{u} \mathrm{r}_{3} \longleftarrow$ $v_{3}, v_{3} \in[\mathrm{m}+1]=\{0,1, \ldots, \mathrm{m}\}, \mathrm{m} \in \mathrm{Z}^{+}(\mathrm{iv}){ }^{s} \mathrm{r}_{1} \longleftrightarrow v_{1}, v_{1} \in[\mathrm{q}+1]=\{0$, $1, \ldots, \mathrm{q}\}, \mathrm{q} \in \mathrm{Z}^{+} ;(\mathrm{v}){ }^{s} \mathrm{r}_{2} \longleftarrow v_{2}, v_{2} \in[\mathrm{y}+1]=\{0,1, \ldots, \mathrm{y}\}, \mathrm{y} \in \ddot{A}$, where $\ddot{\mathrm{A}}$ is a letteral alphabet; (vi) $\left.{ }^{s} \mathrm{r}_{3} \longleftarrow v_{3}, v_{3} \in[\mathrm{n}+1]=\{0,1, \ldots, \mathrm{n}\}, \mathrm{n} \in \mathrm{Z}^{+}\right\}$.

S08 Point $\left(\mathrm{A} \longleftarrow \mathrm{x}=\left\langle\mathrm{r}_{3} \longleftarrow \nu \bmod (\mathrm{m}+1), \mathrm{r}_{2} \longleftarrow \mathrm{A}, \mathrm{r}_{1} \longleftarrow \mathrm{v} \bmod (\mathrm{q}+\right.\right.$ 1) $\left.: \mathrm{r}_{u}\right\rangle: \mathrm{C}$ ) points $\mathrm{A}$ by a ray of light called $\mathrm{x}$ that does carry the loaded information $\left\langle\mathrm{r}_{3} \longleftarrow \nu \bmod 6, \mathrm{r}_{2} \longleftarrow \mathrm{A}, \mathrm{r}_{1} \longleftarrow \nu \bmod 4: \mathrm{r}_{u}\right\rangle$. Hence, one has $\mathrm{C} \longleftarrow(\mathrm{A} \longleftarrow$ $\left.\mathrm{x}=\left\langle\mathrm{r}_{3}, \mathrm{r}_{2}, \mathrm{r}_{1}: \mathrm{r}_{u}\right\rangle, \mathrm{B} \longleftarrow \mathrm{y}=\left\langle\mathrm{r}_{1}, \mathrm{r}_{2}, \mathrm{r}_{3}: \mathrm{r}_{s}\right\rangle\right)$ implies that $\mathrm{C} \longleftarrow\left(\mathrm{A} \longleftarrow\left\langle\mathrm{r}_{3}, \mathrm{r}_{2}\right.\right.$,

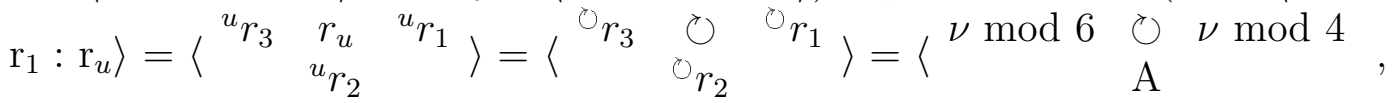




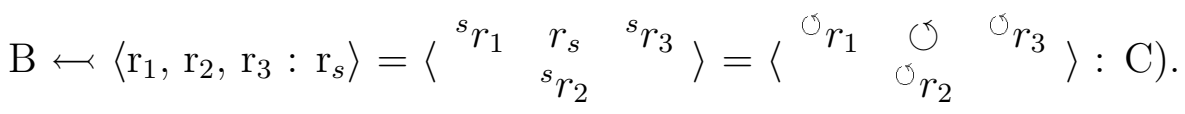

S09 Point $\left(\mathrm{B} \hookleftarrow \mathrm{y}=\left\langle\mathrm{r}_{1} \longleftarrow \nu \bmod 5, \mathrm{r}_{2} \longleftarrow \mathrm{B}, \mathrm{r}_{3} \longleftarrow \nu \bmod 8: \mathrm{r}_{s}\right\rangle: \mathrm{C}\right)$ points $\mathrm{B}$ by a ray of light called $\mathrm{y}$ that does carry the loaded information $\left\langle\mathrm{r}_{1}\right.$ $\left.\longleftarrow \nu \bmod 6, \mathrm{r}_{2} \longleftarrow \mathrm{B}, \mathrm{r}_{3} \longleftarrow \nu \bmod 8: \mathrm{r}_{u}\right\rangle$. Hence, one has $\mathrm{C} \longleftarrow\left(\mathrm{A} \longleftarrow \mathrm{x}=\left\langle\mathrm{r}_{3}\right.\right.$, $\left.\left.\mathrm{r}_{2}, \mathrm{r}_{1}: \mathrm{r}_{u}\right\rangle, \mathrm{B} \longleftarrow \mathrm{y}=\left\langle\mathrm{r}_{1}, \mathrm{r}_{2}, \mathrm{r}_{3}: \mathrm{r}_{s}\right\rangle\right)$ implies that $\mathrm{C} \longleftarrow\left(\mathrm{A} \longleftarrow\left\langle\mathrm{r}_{3}, \mathrm{r}_{2}, \mathrm{r}_{1}: \mathrm{r}_{u}\right\rangle=\right.$

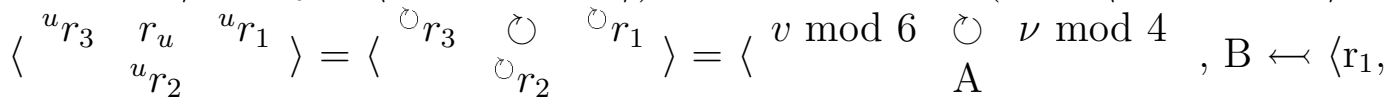

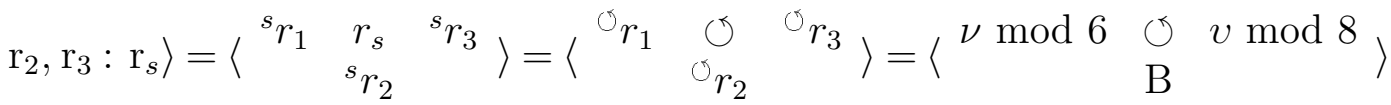
: C).

S10 MakeDesign(A, B: C) makes design of (A, B) in C. Hence one has $\mathrm{C} \longleftarrow\left(\mathrm{A} \longleftarrow \mathrm{x}=\left\langle\mathrm{r}_{3}, \mathrm{r}_{2}, \mathrm{r}_{1}: \mathrm{r}_{u}\right\rangle, \mathrm{B} \longleftarrow \mathrm{y}=\left\langle\mathrm{r}_{1}, \mathrm{r}_{2}, \mathrm{r}_{3}: \mathrm{r}_{s}\right\rangle\right)$ implies

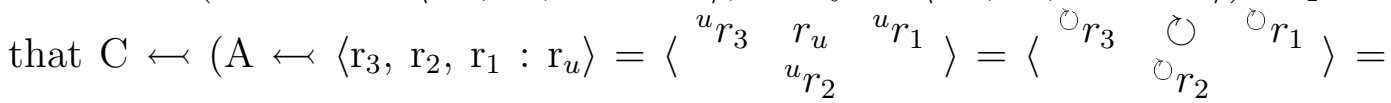
$\left\langle\begin{array}{ccc}5 \bmod 6 & \circlearrowright & 3 \bmod 4 \\ \mathrm{~A} & \end{array}, \mathrm{~B} \longleftarrow\left\langle\mathrm{r}_{1}, \mathrm{r}_{2}, \mathrm{r}_{3}: \mathrm{r}_{s}\right\rangle=\left\langle{ }^{s} r_{1}{ } r_{s}{ }^{s} r_{3}\right\rangle\right.$

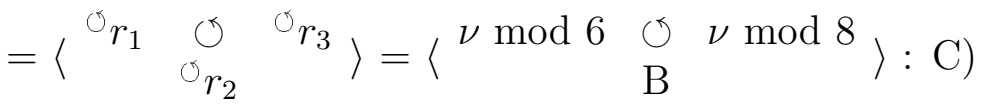

$$
\begin{aligned}
& =\left(\left\langle\begin{array}{lll}
\nu \bmod 6 & \circlearrowright & \nu \bmod 4
\end{array}\right\rangle,\left\langle\begin{array}{lll}
\nu \bmod 6 & \circlearrowleft & 0 \bmod 8
\end{array}\right\rangle: C\right) .
\end{aligned}
$$

S11 ShowDesignof(A, B: M; x, y) gives the designs of A and B like

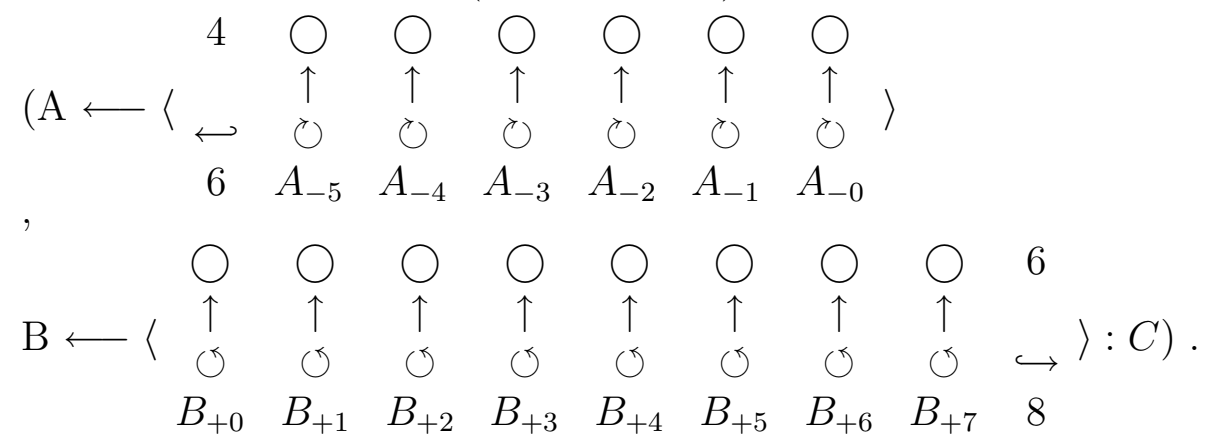

S12 Point(A: M) shows A in $M$ as in S11.

S13 BeamForLoad $(\mathrm{A}: \mathrm{M}, \mathrm{x} \longleftarrow<133102>\bmod 4$ ) registers ( or stores) $\mathrm{x}$ into $\mathrm{A}$ in $\mathrm{M}$. Hence $\mathrm{A}$ becomes like

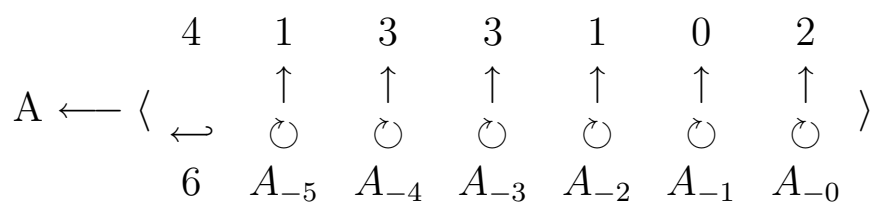

S14 Point(B: M) shows B in $\mathrm{M}$ as in $\mathrm{S} 11$.

S15 BeamForLoad(B: M; y $\longleftarrow<52022534>\bmod 6$ ) registers ( or stores) $\mathrm{y}$ into $\mathrm{B}$ in $\mathrm{M}$. Hence $\mathrm{B}$ becomes like 


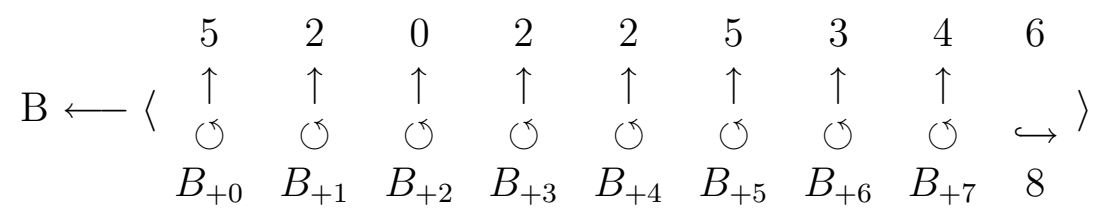

S16 ShowBeamof (A, B: C) Shows $<133102>\bmod 4$ in A and <52022534> $\bmod 6$ in B. Hence we have:

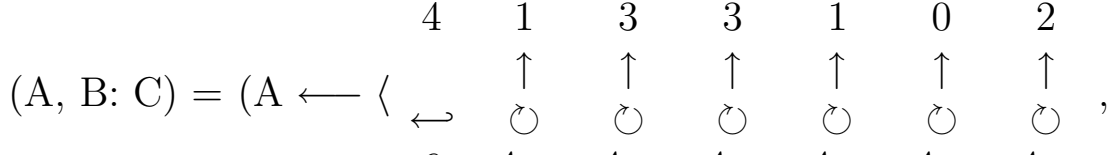

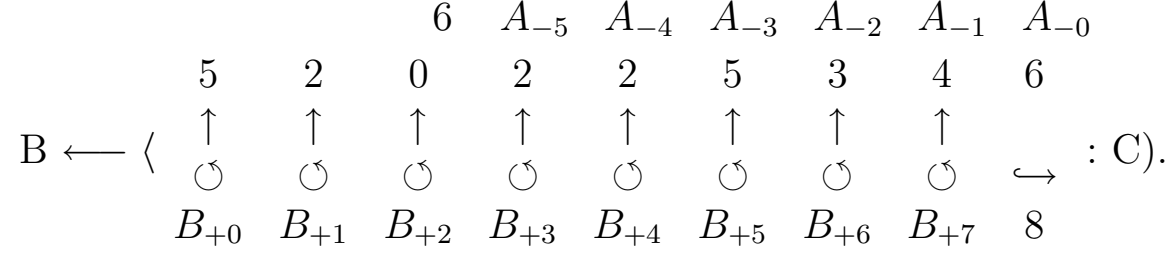

$$
\begin{aligned}
& \text { S17 Stop ; } \\
& \text { S18 End. }
\end{aligned}
$$

(b) Algorithm R2 Let x and y be plemwanal words. Let the conditions in the hypothesis exist for the current use. S00 Begin; S01 Reserve(M: a KBOplem memory cluster); S02 Inject( $(\hat{U})$ into M; Inject $(C)$ into $\hat{U} ; \operatorname{Inject}(A, B)$ into C; S03 Point (A); $\mathrm{A} \longleftarrow \mathrm{x}=\left\langle\mathrm{r}_{3}, \mathrm{r}_{2}, \mathrm{r}_{1}: \mathrm{r}_{u}\right\rangle\left\langle\mathrm{r}_{1}, \mathrm{r}_{2}, \mathrm{r}_{3}: \mathrm{r}_{s}\right\rangle\left\langle\mathrm{r}_{3}, \mathrm{r}_{2}, \mathrm{r}_{1}\right.$ $\left.: \mathrm{r}_{u}\right\rangle ; \operatorname{S04} \operatorname{Point}(\mathrm{B}) ; \mathrm{B} \longleftarrow \mathrm{y}=\left\langle\mathrm{r}_{1}, \mathrm{r}_{2}, \mathrm{r}_{3}: \mathrm{r}_{s}\right\rangle\left\langle\mathrm{r}_{1}, \mathrm{r}_{2}, \mathrm{r}_{3}: \mathrm{r}_{s}\right\rangle\left\langle\mathrm{r}_{3}, \mathrm{r}_{2}, \mathrm{r}_{1}\right.$ : $\left.\mathrm{r}_{u}\right\rangle\left\langle\mathrm{r}_{1}, \mathrm{r}_{2}, \mathrm{r}_{3}: \mathrm{r}_{s}\right\rangle\left\langle\mathrm{r}_{3}, \mathrm{r}_{2}, \mathrm{r}_{1}: \mathrm{r}_{u}\right\rangle ; \mathrm{S} 05$ ShowConfigurationof(A, B); S06 $\operatorname{Point}(\mathrm{A}) ; \mathrm{A} \longleftarrow \mathrm{x}=\left\langle\mathrm{r}_{3} \longleftarrow 5, \mathrm{r}_{2} \longleftarrow \mathrm{A}, \mathrm{r}_{1} \longleftarrow 3: \mathrm{r}_{u}\right\rangle\left\langle\mathrm{r}_{1} \longleftarrow 5, \mathrm{r}_{2} \longleftarrow \mathrm{A}, \mathrm{r}_{3} \longleftarrow\right.$ $\left.2: \mathrm{r}_{s}\right\rangle\left\langle\mathrm{r}_{3} \longleftarrow 5, \mathrm{r}_{2} \longleftarrow \mathrm{A}, \mathrm{r}_{1} \longleftarrow 4: \mathrm{r}_{u}\right\rangle ; \operatorname{Point}(\mathrm{B}) ; \mathrm{B} \longleftarrow \mathrm{y}=\left\langle\mathrm{r}_{1} \longleftarrow 5, \mathrm{r}_{2} \longleftarrow\right.$ $\left.\mathrm{B}, \mathrm{r}_{3} \longleftarrow 2: \mathrm{r}_{s}\right\rangle\left\langle\mathrm{r}_{1} \longleftarrow 3, \mathrm{r}_{2} \longleftarrow \mathrm{B}, \mathrm{r}_{3} \longleftarrow 2: \mathrm{r}_{s}\right\rangle\left\langle\mathrm{r}_{3} \longleftarrow 2, \mathrm{r}_{2} \longleftarrow \mathrm{B}, \mathrm{r}_{1} \longleftarrow 2: \mathrm{r}_{u}\right.$ \rangle$\left\langle\mathrm{r}_{1} \longleftarrow 2, \mathrm{r}_{2} \longleftarrow \mathrm{B}, \mathrm{r}_{3} \longleftarrow 2: \mathrm{r}_{s}\right\rangle ; \operatorname{Design}(\mathrm{A}, \mathrm{B}) ; \operatorname{S} 07 \operatorname{ShowDesignof}(\mathrm{A}, \mathrm{B})$ ; S08 Point(A) ; Beam(A); Point(B); Beam(B) ; S09 ShowBeamof(A, B) ; S10 Point(A, B) ; Load B) ; S11 ShowLoadedof(A, B) ; S12 Stop ; S13 End.

(c) Algorithm R3 Let 1) $\mathrm{w}_{1}=\left\langle\mathrm{r}_{3}, \mathrm{r}_{2}, \mathrm{r}_{1}: \mathrm{r}_{u}\right\rangle\left\langle\mathrm{r}_{1}, \mathrm{r}_{2}, \mathrm{r}_{3}: \mathrm{r}_{s}\right\rangle\left\langle\mathrm{r}_{3}, \mathrm{r}_{2}\right.$, $\left.\mathrm{r}_{1}: \mathrm{r}_{u}\right\rangle$ and $\mathrm{w}_{2}=\left\langle\mathrm{r}_{1}, \mathrm{r}_{2}, \mathrm{r}_{3}: \mathrm{r}_{s}\right\rangle\left\langle\mathrm{r}_{1}, \mathrm{r}_{2}, \mathrm{r}_{3}: \mathrm{r}_{s}\right\rangle\left\langle\mathrm{r}_{3}, \mathrm{r}_{2}, \mathrm{r}_{1}: \mathrm{r}_{u}\right\rangle\left\langle\mathrm{r}_{1}, \mathrm{r}_{2}\right.$, $\left.\mathrm{r}_{3}: \mathrm{r}_{s}\right\rangle$ be two plemwanal words; 2) $\mathrm{L}_{1}=\left\{\mathrm{w}_{1}\right\}$ and $\mathrm{L}_{2}=\left\{\mathrm{w}_{1}, \mathrm{w}_{2}\right\}$ be two plemwanals. S00 Begin; S01 Reserve(M: a KBO-plem memory cluster); S02 $\operatorname{Inject}(\hat{U})$ into $M$; Inject $(C)$ into $\hat{U} ; \operatorname{Inject}(A, B)$ into $C$; S03 Point $(A) ; A$ $\longleftrightarrow \mathrm{w}_{1}=\left\langle\mathrm{r}_{3}, \mathrm{r}_{2}, \mathrm{r}_{1}: \mathrm{r}_{u}\right\rangle\left\langle\mathrm{r}_{1}, \mathrm{r}_{2}, \mathrm{r}_{3}: \mathrm{r}_{s}\right\rangle\left\langle\mathrm{r}_{3}, \mathrm{r}_{2}, \mathrm{r}_{1}: \mathrm{r}_{u}\right\rangle ; \mathrm{S} 04 \operatorname{Point}(\mathrm{B}) ; \mathrm{B} \longleftarrow$ $\mathrm{w}_{2}=\left\langle\mathrm{r}_{1}, \mathrm{r}_{2}, \mathrm{r}_{3}: \mathrm{r}_{s}\right\rangle\left\langle\mathrm{r}_{1}, \mathrm{r}_{2}, \mathrm{r}_{3}: \mathrm{r}_{s}\right\rangle\left\langle\mathrm{r}_{3}, \mathrm{r}_{2}, \mathrm{r}_{1}: \mathrm{r}_{u}\right\rangle\left\langle\mathrm{r}_{1}, \mathrm{r}_{2}, \mathrm{r}_{3}: \mathrm{r}_{s}\right\rangle\left\langle\mathrm{r}_{3}, \mathrm{r}_{2}\right.$, $\left.\mathrm{r}_{1}: \mathrm{r}_{u}\right\rangle ; \mathrm{L}_{1} \longleftarrow\{\mathrm{A}\} ; \mathrm{L}_{2} \longleftrightarrow\{\mathrm{A}, \mathrm{B}\} ; \mathrm{S} 05$ ShowConfigurationof $\left(\mathrm{L}_{1}, \mathrm{~L}_{2}\right) ; \mathrm{S} 06$ $\operatorname{Point}(\mathrm{A}) ; \mathrm{A} \longleftarrow\left\langle\mathrm{r}_{3} \longleftarrow 5, \mathrm{r}_{2} \longleftarrow \mathrm{A}, \mathrm{r}_{1} \longleftarrow 3: \mathrm{r}_{u}\right\rangle\left\langle\mathrm{r}_{1} \longleftarrow 5, \mathrm{r}_{2} \longleftarrow \mathrm{A}, \mathrm{r}_{3} \longleftarrow\right.$ $\left.2: \mathrm{r}_{s}\right\rangle\left\langle\mathrm{r}_{3} \longleftarrow 5, \mathrm{r}_{2} \longleftarrow \mathrm{A}, \mathrm{r}_{1} \longleftarrow 4: \mathrm{r}_{u}\right\rangle ; \operatorname{Point}(\mathrm{B}) ; \mathrm{B} \longleftarrow\left\langle\mathrm{r}_{1} \longleftarrow 5, \mathrm{r}_{2} \longleftarrow \mathrm{B}\right.$, $\left.\mathrm{r}_{3} \longleftarrow 2: \mathrm{r}_{s}\right\rangle\left\langle\mathrm{r}_{1} \longleftarrow 3, \mathrm{r}_{2} \longleftarrow \mathrm{B}, \mathrm{r}_{3} \longleftarrow 2: \mathrm{r}_{s}\right\rangle\left\langle\mathrm{r}_{3} \longleftarrow 2, \mathrm{r}_{2} \longleftarrow \mathrm{B}, \mathrm{r}_{1} \longleftarrow 2: \mathrm{r}_{u}\right\rangle\langle$ $\left.\mathrm{r}_{1} \longleftarrow 2, \mathrm{r}_{2} \longleftarrow \mathrm{B}, \mathrm{r}_{3} \longleftarrow 2: \mathrm{r}_{s}\right\rangle ; \operatorname{Design}(\mathrm{A}, \mathrm{B}) ; \mathrm{L}_{1} \longleftarrow\{\mathrm{A}\} ; \mathrm{L}_{2} \longleftarrow\{\mathrm{A}, \mathrm{B}\} ; \mathrm{S} 07$ 
ShowDesignof $\left(\mathrm{L}_{1}, \mathrm{~L}_{2}\right) ; \operatorname{S} 08 \operatorname{Point}(\mathrm{A}) ; \operatorname{Beam}(\mathrm{A}) ; \operatorname{Point}(\mathrm{B}) ; \operatorname{Beam}(\mathrm{B})$; $\mathrm{L}_{1} \longleftarrow\{\mathrm{A}\} ; \mathrm{L}_{2} \longleftrightarrow\{\mathrm{A}, \mathrm{B}\} ; \operatorname{S} 09 \operatorname{ShowBeamof}\left(\mathrm{L}_{1}, \mathrm{~L}_{2}\right) ; \operatorname{S10} \operatorname{Point}(\mathrm{A}, \mathrm{B}) ;$ $\operatorname{Load}(\mathrm{A}, \mathrm{B}) ; \mathrm{L}_{1} \longleftrightarrow\{\mathrm{A}\} ; \mathrm{L}_{2} \longleftrightarrow\{\mathrm{A}, \mathrm{B}\} ; \mathrm{S} 11 \operatorname{ShowLoaded}\left(\mathrm{L}_{1}, \mathrm{~L}_{2}\right) ; \mathrm{S} 12$ $\operatorname{Point}(\mathrm{A}, \mathrm{B}) ; \boldsymbol{F i x}(\mathrm{A}, \mathrm{B}) ; \mathrm{L}_{1} \longleftarrow\{\mathrm{A}\} ; \mathrm{L}_{2} \longleftrightarrow\{\mathrm{A}, \mathrm{B}\} ; \mathrm{S} 13 \operatorname{ShowFixed}\left(\mathrm{L}_{1}, \mathrm{~L}_{2}\right.$ ) ; S14 Stop ; S15 End.

\section{$5 \quad$ Main Results}

This paper develops, studies and introduces us-crop based plemwanal. It is a formal language type in which numerical words with communicating antennae produce a special computer mathematics generator type of machine KBO. It has a self communication process in the overall process of organizing token classical or token quantum substructures. Plemal in this paper has been introduced by 10 definitions, 2 corollaries and one theorem for covering the ideas of configurational KBO types and remote programming style. Author claims that plemwanal is a very special communicating computer mathematics generating language type. It is produced as an extension of plemal for obtaining an artificial intelligently communicating numerical words with antennae. These numerical words with antennae are communicating themselves by broadcasting and intercepting information in the execution time of its substructures. Each token numerical word with antennae in each substructure has wireless communication property with each other and with its environment. It is designed for generating remote, parallel, sequential, mobile, distributed, etc. type us-crop based compact I@I KBO cluster plem memory programming in a classical or quantum information processing environment. After plemwanal there is going to be a new computing mathematics type. The author calls it as "Communicating Computer Mathematics."

\section{References}

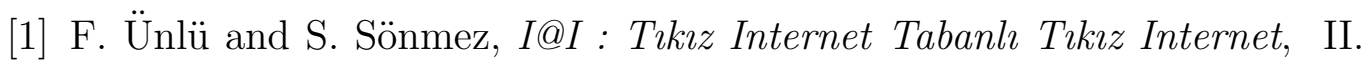
Proceeding of Information Technology Congress, pp. 246-248, 1-May 2003, Pamukkale University-Denizli, Turkey.

[2] F. Ünlü and S. Sönmez, Convoluted Prüfer's Type I@I, International Mathematical Journal, Vol. 4, no. 6, pp. 539 - 547, 2003.

[3] F. Ünlü, An Intuitive Differential Equation Model for Knowledge Based Objects(KBO) Representation of Science, ISCISXIV, Proceeding of The Fourteenth International Symposium on Computer and Information Science, pp1066-1068, October, 18-20, Kuşadası, Aydın, Turkey, 1999. 
[4] F. Ünlü, FTD Grammar Graphs, International Journal of Computer Mathematics Vol. 80, no. 1, pp1-9, January 2003.

[5] F. Ünlü, Chance Constrained Threshold KBO System Design, International Mathematical Journal, Vol. 5, no. 4, pp 321 - 328, 2004.

[6] F. Ünlü, A Generalized us-Culture Job Scheduling for Forecasting Problems, International Mathematical Journal, Vol. 4, no. 4, pp 313-320, 2003.

[7] F. Ünlü, S. Sönmez, and Z. I. Ünlü, Linear Circular Convoluted I@I KBO Cluster Generating us-Crop, International Mathematical Journal, Vol. 5, no. 4, pp 329 - 338, 2004.

[8] F. Ünlü, tuze-Channeled KBO for DCP, International Mathematical Journal, Vol. 5. no. 4, pp 339 - 346, 2004.

[9] F. Ünlü, The Fundamental Mathematics of us-Crop Based Compact plem Memory, (unpublished).

[10] F. Ünlü, The Fundamental Mathematics of Plemal, (unpublished).

[11] S. Washburn, T. Marlowe and C. T. Ryan, Discrete Mathematics, AddsonWesley, New York, 2000.

[12] J. D. Baum, Elements of Point Set Topology, Prentice-Hall, Inc., Engelwood Cliffs, N.J., 1964.

[13] R. P. Grimald,, Discrete and Combinatorial Mathematics, AddisonWesley, New York, 1999.

Received: October 25, 2005 